\title{
Nuclear Features in the Diagnosis of Follicular Variant of Papillary Thyroid Carcinoma-The Diagnostic Dilemma
}

\author{
Chaganti Padmavathi Devi', Karri Maruthi Devi', Madabhushi Venugopal', \\ Mulukutla Partha Akarsh'2 \\ ${ }^{1}$ Guntur Medical College, Guntur, India \\ ${ }^{2}$ Siddhartha Medical College, Vijayawada, India \\ Email: drcpd60@gmail.com, ratnakumardevi@gmail.com, mvgopal@yahoo.com, akarsh316@yahoo.com
}

Received 10 April 2015; accepted 24 July 2015; published 27 July 2015

Copyright (C) 2015 by authors and Scientific Research Publishing Inc.

This work is licensed under the Creative Commons Attribution International License (CC BY).

http://creativecommons.org/licenses/by/4.0/

(c) (i) Open Access

\begin{abstract}
Aim: Follicular variant of papillary thyroid carcinoma [FVPC] as a diagnostic entity has been beset by many controversies. In this study, we describe the nuclear features essential for the diagnosis and analyze the difficulties that confront pathologists as it is important to avoid pitfalls because appropriate management protocol depends upon on an accurate diagnosis of this variant. Materials and Methods: A total of 30 cases, diagnosed as FVPC over a period of two years in the Department of Pathology, were taken for the study. Haematoxylin and Eosin stained sections were reviewed. The extent and distribution of nuclear features were analyzed. Results: The 30 cases of FVPC were categorized into encapsulated and infiltrative groups basing on the presence or lack of capsule and capsular invasion and vascular invasion. Conclusion: FVPC is diagnosed basing on specific nuclear features and hence histopathology still remains the gold standard for the accurate diagnosis.
\end{abstract}

\section{Keywords}

Nuclear Features, Follicular Variant, Papillary Carcinoma, Encapsulation, Vascular Invasion Capsular Invasion

\section{Introduction}

Papillary thyroid carcinoma [PTC] is conventionally diagnosed basing on cytological features rather than archi-

How to cite this paper: Devi, C.P., Devi, K.M., Venugopal, M. and Akarsh, M.P. (2015) Nuclear Features in the Diagnosis of Follicular Variant of Papillary Thyroid Carcinoma-The Diagnostic Dilemma. International Journal of Otolaryngology and Head \& Neck Surgery, 4, 296-302. http://dx.doi.org/10.4236/ijohns.2015.44051 
tectural pattern whereas Follicular carcinoma of thyroid is diagnosed basing on the presence of invasion [1]. Lindsay [2] first described the entity of follicular variant of papillary thyroid carcinoma (FVPC) which was later defined by Chen and Rosai [3]. During the last three decades either infiltrative or encapsulated thyroid tumors with follicular pattern were diagnosed as papillary carcinoma basing on the nuclear features like enlargement, elongation, nuclear clearing, intranuclear grooves, inclusions, micronucleoli and thick nuclear membranes [4] [5]. However, problems cropped up in the diagnosis of completely encapsulated tumors because of variability among pathologists due to the lack of agreement on the minimal diagnostic criteria [4] [6]. Chan proposed criteria for the diagnosis of encapsulated FVPC which include clear nuclei, round to oval nuclei, crowded nuclei, intranuclear grooves, psammoma bodies and secondary features like irregular follicles, thick colloid and multinucleated histiocytes [1]. A scoring system was applied for the diagnosis of PTC by Verhulst et al. who observed that follicular patterned PTC was at or below the threshold score [7].

The inter- and intra-observer variability often leads to confusion among clinicians regarding the management of FVPC. To overcome this grey zone, the term "well differentiated thyroid tumor of uncertain malignant potential" was coined by Chernobyl Pathologist group for encapsulated follicular patterned tumor with incomplete or unconvincing features of PTC [8]. Livolsi VA proposed that FVPTC is a heterogeneous group and defined 6 histologic subtypes basing on variation in architectural pattern and distribution of nuclear features [9].

Hung-Yu Chang et al. found that, though the clinical behavior of FVPC hovers between that of pure papillary carcinoma and follicular carcinoma, prognosis following aggressive treatment was similar to pure papillary cancer [10]. As such a thorough and diligent scrutiny of sections sampled from representative areas and strict adherence to the specific criteria are imperative for an accurate diagnosis of FVPC which is of paramount importance in planning of an appropriate and personalized treatment protocol.

Aim: This study is undertaken to reclassify FVPC basing on architectural and cytological features and discuss the spectrum of nuclear features in the diagnosis of FVPC and the difficulties encountered in the evaluation of subtypes of FVPC.

\section{Material and Methods}

In this study the tumors which were diagnosed as FVPC between January, 2011 and December, 2012 were taken. The cases included total thyroidectomy or hemithyroidectomy surgical specimens. Tumor size was measured in the greatest diameter. Surgical margins were also inked. The sections were stained with hematoxylin and eosin.

Exclusion Criteria-Tumors with presence of necrosis and increased mitotic activity of more than 5 per $10 \mathrm{HPF}$ were excluded from the present study. Tumors showing papillary configurations constituting more than $1 \%$ of tumor area were excluded from the diagnosis of FVPC [4].

\section{Results}

A total of 30 cases diagnosed as FVPC reported during the period of two years were selected for the present study. The cases were subdivided in to encapsulated and infiltrative tumors. We classified them as encapsulated if the tumor was completely surrounded by a capsule and infiltrative if there was no capsule or incomplete capsulation with tongues of tumor tissue infiltrates in to non-neoplastic thyroid. Tumors that were grossly encapsulated with no microscopic evidence of capsular and vascular invasion were designated as noninvasive encapsulated FVPC. Tumors that were grossly encapsulated but showing microscopic evidence of capsular or vascular infiltration were classified as invasive encapsulated (Figure 1).

In our study twenty seven cases were found to be encapsulated tumors while the remaining three cases showed infiltrative features. Encapsulated tumors were again subdivided in to noninvasive and invasive groups basing on absence or presence of capsular and vascular invasion. Non invasive encapsulated tumors were 21 in number where as the remaining six were identified as invasive encapsulated tumors.

Complete infiltration of entire thickness of tumor capsule is taken as capsular infiltration (Figure 2). However tumor nests embedded within the tumor capsule and irregularity of the contour along the inner border of the capsule were not considered as evidence of capsular invasion. Vascular invasion implies invasion of a vessel with in or out side tumor capsule with covering by endothelium or attachment to the vessel wall [3] (Figure 3).

In our study women outnumbered men and the commonest age group was 20 to 30 years.

The size of the tumor in our cases is less than $4 \mathrm{cms}$ in 28 cases and more than $4 \mathrm{cms}$ in 2 cases. 


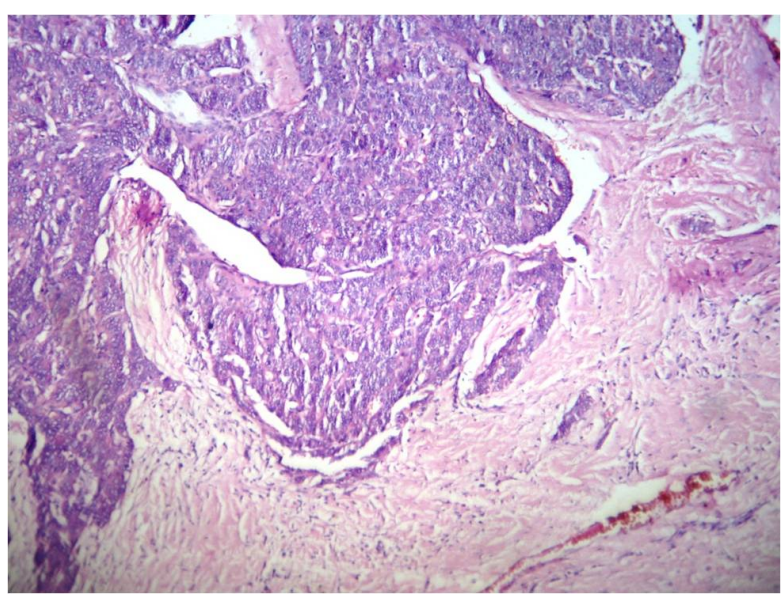

Figure 1. Infiltrative tumor.

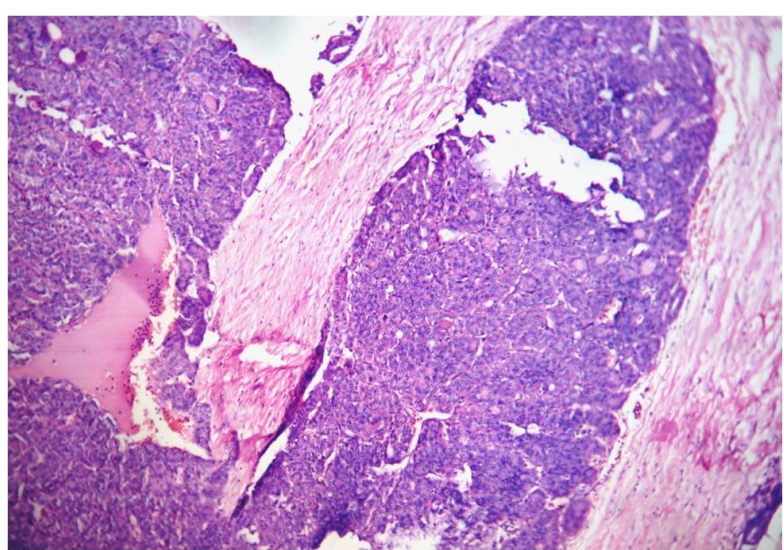

Figure 2. Full thickness capsular infiltration.

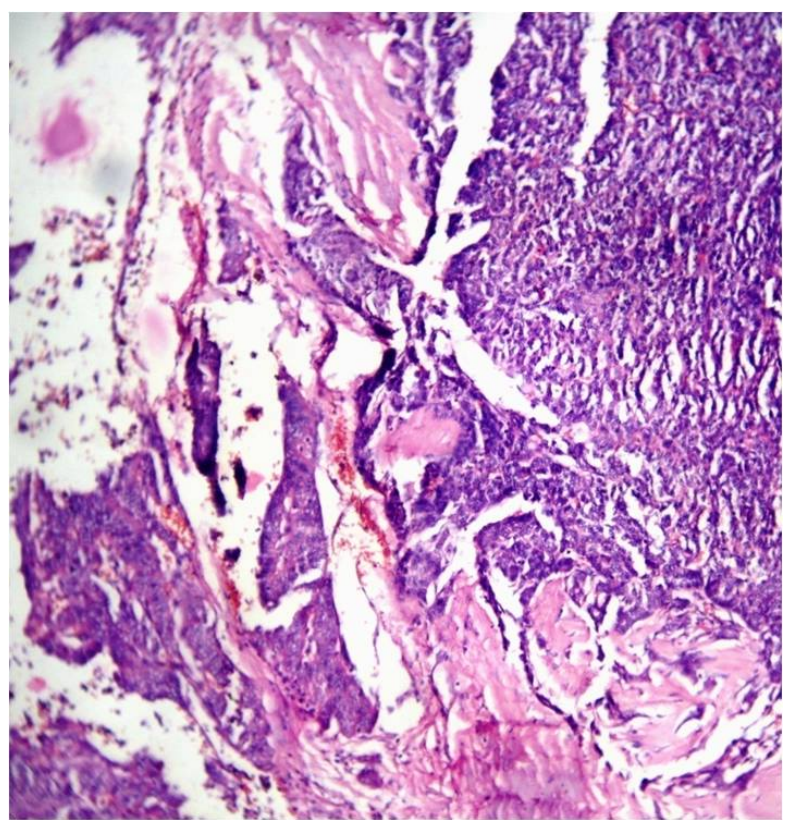

Figure 3. Vascular infiltration-Tumor attached to the wall of the blood vessal. 
The nuclear and other morphological features which favored the diagnosis of FVPC in the descending order of frequency in our cases were pale nuclei, crowding of nucleus (Figure 4), thick colloid (Figure 5), nuclear grooves, round to oval nuclei, psammoma bodies and multicentricity.

\section{Discussion}

The differential diagnosis of thyroid neoplasms with follicular architecture includes adenomatous hyperplasias, Follicular Adenoma [FA], Follicular Carcinoma [FC] and FVPC. Tumors which were predominantly composed of follicles with nuclear features of PTC were classified under the category of FVPC [11].

In the study of Liu Jeffery, the median age of the patients was 43.1 years and females outnumbered males and constituted $76 \%$ of cases [12]. In our study also females outnumbered males and most cases were seen in third decade.

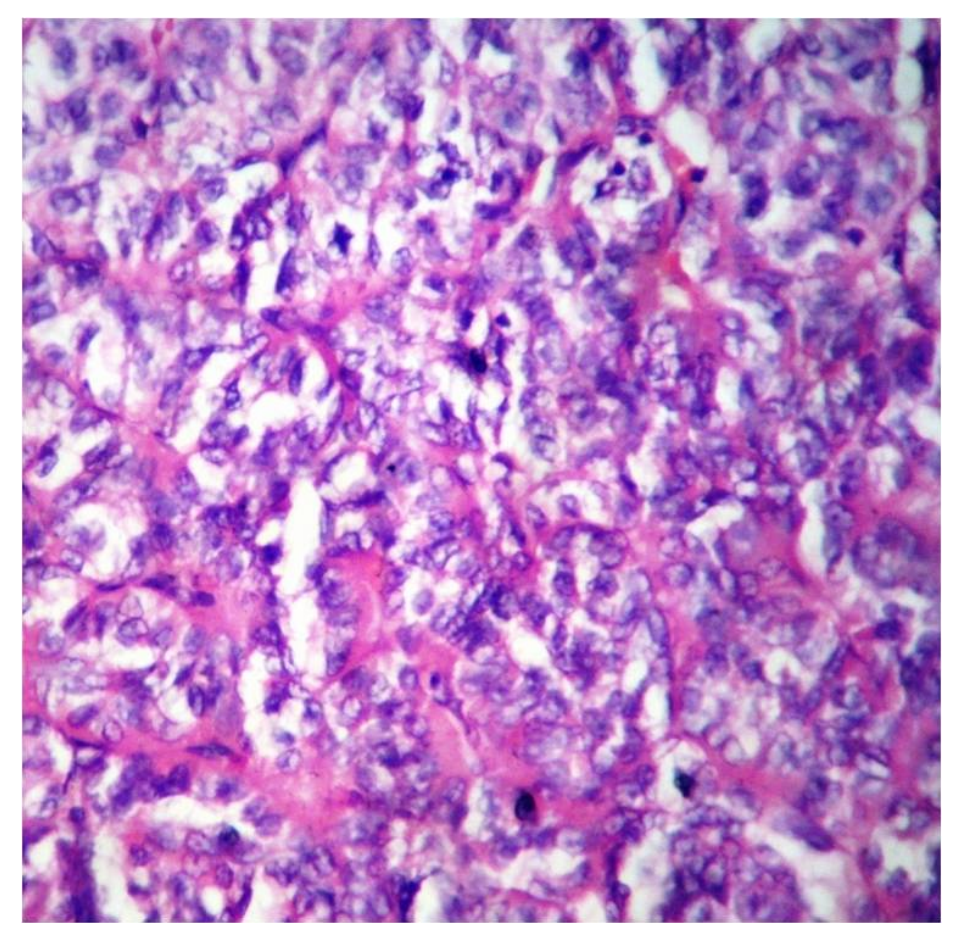

Figure 4. Pale nuclei,oval nuclei and crowding of nuclei.

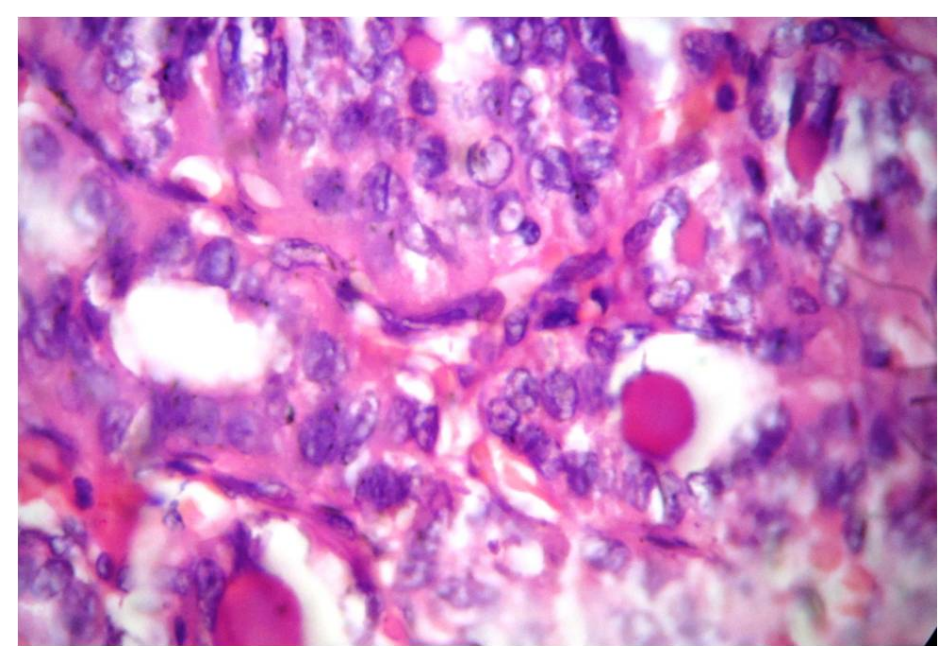

Figure 5. Nuclear grooves and thick colloid. 
FVPC clinically, histologically and on molecular basis are divided into two distinct groups. The first group is encapsulated and the second one is non-encapsulated which includes infiltrative and diffuse forms [12]. The infiltrative growth resembles classic PTC in its invasive growth and metastasis to lymph nodes. FVPC has more favorable clinicopathological features in the form of frequent encapsulation, lower rates of lymph node metastases and less frequent extra thyroidal extension [13] [14]. However, some encapsulated FVPC behave like follicular neoplasm and may even metastasize to distant sites [11]. In the studies of Chen and Rosai [3] and Jeffery Liu [12] encapsulated FVPC accounted for more number of tumors than infiltrative type. In our study also encapsulated tumors outnumbered infiltrative tumors. This finding is in concordance with the world literature.

Nuclear features: FVPC can be easily diagnosed when nuclear features of papillary carcinoma are classic and diffuse. However the diagnosis of FVPC becomes very difficult if the nuclear features are not well developed and when they are focally present [4].

Some authors believe that there is an increase in the reporting of FVPC because of lowering of thresholds [4]. Renshaw et al. observed that lack of minimum histological definition and reports of rare aggressive clinical behavior resulted in a marked lowering of diagnostic threshold of FVPC and consequent over diagnosis of this entity [15]. Elsheikh TM et al. noted that there are no strict guidelines regarding the quantitative criteria to diagnose FVPC including the percentage of tumor showing nuclear features of papillary carcinoma [NFPC], the degree of nuclear pallor, the number of nuclear grooves and optically clear nuclei [4].

According to Livolsi et al. strict criteria for specific nuclear features comprising of ovoid nuclei, crowded nuclei, clear nuclei, intranuclear grooves and inclusions should be applied to diagnose FVPC [7]. They also observed that other favorable features included psammoma bodies and secondary features such as elongated irregularly shaped follicles, dark staining colloid, and multinucleated histiocytes in the lumen of the follicles [5]. In the decreasing order of preference the histological features taken in to consideration for the diagnosis of FVPC are cytoplasmic invaginations, abundant nuclear grooves, ground glass nuclei, psammoma bodies, enlarged overlapping nuclei, and irregularly shaped nuclei [5].

In the study of Tarik M. Elsheikh et al., nuclear features in the decreasing order of frequency were nuclear clearing, very fine powdery chromatin, nuclear grooves, nuclear overlapping, nuclear membrane irregularity, and nuclear enlargement [4]. The secondary features comprised chromatin margination, distorted follicular architecture and fibrosis/sclerosis [4]. According to Chan JK the features necessary to make the diagnosis of FVPC include ovoid nuclei rather than round nuclei, crowded nuclei with lack of polarization, nuclei which show pale or clear chromatin pattern with the nuclear clearing not to be confined to the centre, nuclear grooves and psammoma bodies [1]. The additional features that should be taken in to consideration, if the above features were lacking, include abortive papillae, elongated or irregularly shaped follicles, dark staining colloid, presence of rare and multinucleated histiocytes in the lumen of the follicles.

In our study features seen in the decreasing order of frequency were pale nuclei, nuclear grooves, abortive papillae, nuclear pseudo inclusions, nuclear clearing, followed by crowded nucleus, Psammoma bodies and thick colloid. Sclerosis was consistently seen in all invasive cases.

Rosai et al. subscribe to the view that ground glass nuclei should be seen in most areas to diagnose a lesion as PTC. Plain vesicular nuclei are seen both in benign and malignant thyroid lesions and hence should not be taken as diagnostic marker for FVPC [16].

In view of the various morphological presentations of FVPC and controversies Virginia A Livolsi proposed that FVPC is a heterogeneous group and classified them into six variants [9].

Type 1: Unencapsulated invasive tumor where the lesion grows in infiltrative pattern [16].

Type 2: Encapsulated invasive tumors with diffuse classical features

Type 3: Encapsulated invasive tumors showing either classical or unconvincing nuclear features which are present in multifocal areas

Type 4: Encapsulated noninvasive tumors with classical and diffuse nuclear features

Type 5: Encapsulated noninvasive tumors with classical or unconvincing nuclear features which are present multifocally.

FVPC-Type 2 to 5: They represent a group of capsulated tumors which are invasive or noninvasive. They present diagnostic difficulty when the nuclear features are present in multiple locations in the same tumor. Cases of noninvasive encapsulated variant of FVPC with multifocal but incomplete nuclear features are the ones which caused diagnostic problems and greatest inter observer variability. In such cases, Lioyd et al. and Elsheikh observed that the best developed nuclear features were seen in the rim of tumor closest to the tumor capsule [4] [6]. 
Livolsi also suggests that encapsulated FVPC may represent a hybrid of papillary carcinoma and follicular adenoma or carcinoma [9].

Type 6: Capsulated lesion of thyroid in which subcentimeter area shows classical nuclear features of PTC, the lesion was designated as papillary microcarcinoma in Follicular Adenoma [11]. In our study we did not come across type 6 cases.

Baloch and Livolsi found that encapsulated FVPC with multifocal nuclear features developed distant metastasis [11]. Rivera et al. observed that invasive encapsulated tumors do possess metastatic potential but the sites of secondary deposits vary from the classic papillary cancer. They also found that FVPC without capsular or vascular invasion lacked lymph node deposits and did not recur [17].

Chernobyl Pathologist Group classified neoplasms which showed questionable nuclear features and lack of vascular or capsular invasion as well differentiated tumors of uncertain malignant potential or hybrid tumors [1]. In our study, one noninvasive tumor with unconvincing nuclear features was reported as well differentiated tumor of uncertain malignant potential.

According to Livolsi, even when the nuclear change is multifocal but not diffuse, the entire lesion could be malignant [9]. This possibility is supported by different molecular and immunohistochemical studies [18] [19]. Markers like high molecular weight cytokeratin, cytokeratin 19, HBME1, CD 57, CD 44, and CD 15 are commonly expressed in PTC when compared to non neoplastic lesions of thyroid. But their expression is not sufficient for the diagnosis of FVPC because in classic PTC also they are focally expressed [20]. CD 56 is considered a negative marker for the diagnosis of PTC as it is expressed in $93 \%$ of benign lesions and while it is expressed in only $5 \%$ of cases of PTC [20].

A Salajegheh et al. believe that FVPTC has different molecular changes compared to conventional papillary thyroid carcinoma which lead to phenotypic differences [21]. Adeniran AJ observed that BRAF, RET/PTC, and RAS mutations are associated with distinct microscopic, clinical, and biologic features of thyroid papillary carcinomas and RAS mutations characterize exclusively FVPC and correlate with less prominent nuclear features [22]. Livolsi et al., however, are of the view that the field is still evolving and molecular studies of subtypes of FVPC are required to define these tumors [5].

\section{Conclusion}

Follicular variant of papillary thyroid carcinoma can present as uncapsulated infiltrative variant or partly capsulated or encapsulated variants. The diagnosis of FVPC is made primarily basing on nuclear features. However nuclear features when incomplete or borderline and multifocal can pose diagnostic difficulty. Immunohistochemical markers, either positive or negative, are not 100\% diagnostic of FVPC. Better understanding of molecular basis of FVPC will help in identifying precise markers. As such histopathology still remains the gold standard for the accurate diagnosis of FVPC.

\section{References}

[1] Chan, J.K. (2002) Strict Criteria Should Be Applied in the Diagnosis of Encapsulated Follicular Variant of Papillary Carcinoma of Thyroid. American Journal of Clinical Pathology, 117, 16-18. http://dx.doi.org/10.1309/P7QL-16KQ-QLF4-XW0M

[2] Lindsay, S. (1960) Carcinoma of the Thyroid Gland. A clinical and Pathological Study of 239 Patients at the University of California Hospital. Charles C Thomas, Springfield, IL.

[3] Chen, K.T. and Rosai, J. (1977) Follicular Variant of Thyroid Papillary Carcinoma: A Clinicopathological Study of Six Cases. American Journal of Surgical Pathology, 1, 171-175. http://dx.doi.org/10.1097/00000478-197706000-00003

[4] Elsheikh, T.M., Asa, S.L., Chan, J.K., et al. (2008) Interobserver and Intraobserver Variation among Experts in the Diagnosis of Thyroid Follicular Lesions with Borderline Nuclear Features of Papillary Carcinoma. American Journal of Clinical Pathology, 30, 736-744. http://dx.doi.org/10.1309/AJCPKP2QUVN4RCCP

[5] Livolsi, V.A. and Baloch, Z.W. (2009) The Many Faces of Follicular Variant of Papillary Thyroid Carcinoma. Pathology Case Reviews, 14, 214-218. http://dx.doi.org/10.1097/PCR.0b013e3181c75e9b

[6] Lioyd, R.V., Erickson, L.A., Casey, M.B., et al. (2004) Observer Variation in the Diagnosis of Follicular Variant of Papillary Thyroid Carcinoma. American Journal of Surgical Pathology, 28, 1336-1340. http://dx.doi.org/10.1097/01.pas.0000135519.34847.f6

[7] Verhulst, P., Devos, P., Aubert, S., et al. (2008) A Score Based on Microscopic Criteria Proposed for Analysis of Papillary Carcinoma of Thyroid. Virchows Archiv, 452, 233-240. http://dx.doi.org/10.1007/s00428-008-0577-x 
[8] Williams, E.D., Abrosimov, A., Bogdanova, T.I., et al. (2000) Two Proposals Regarding the Terminology of Thyroid Tumors [Guest Editorial]. International Journal of Surgical Pathology, 8, 181-183. http://dx.doi.org/10.1177/106689690000800304

[9] Livolsi, V.A. and Baloch, Z.W. (2009) The Many Faces of Follicular Variant of Papillary Thyroid Carcinoma. Pathology Case Reviews, 14, 214-218. http://dx.doi.org/10.1097/PCR.0b013e3181c75e9b

[10] Chang, H.-Y., Lin, J.-D., Chou, S.-C., Chao, T.-C. and Hsueh, C. (2006) Clinical Presentations and Outcomes of Surgical Treatment of Follicular Variant of the Papillary Thyroid Carcinomas. Japanese Journal of Clinical Oncology, 36, 688-693. http://dx.doi.org/10.1093/jjco/hyl093

[11] Baloch, Z.W. and Livolsi, V.A. (2002) Follicular-Patterned Lesions of the Thyroid. American Journal of Clinical Pathology, 117, 143-150. http://dx.doi.org/10.1309/8VL9-ECXY-NVMX-2RQF

[12] Liu, J., Singh, B., Tullini, G., Curlson, D.L., et al. (2006) Follicular Variant of Papillary Thyroid Carcinoma: A Clinicopathological Study of a Problematic Entity. Cancer, 6, 1255-1264. http://dx.doi.org/10.1002/cncr.22138

[13] Passler, C., Prager, G., Scheuba, G., et al. (2003) Follicular Variant of Papillary Thyroid Carcinoma: A Long Term Follow-Up. Archives of Surgery, 138, 1362-1366. http://dx.doi.org/10.1001/archsurg.138.12.1362

[14] Zidan, J., Karen, D., Stein, M., et al. (2003) Pure versus Follicular Variant of Papillary Thyroid Carcinoma: Clinical Features, Prognostic Factors, Treatment, and Survival. Cancer, 97, 1181-1185. http://dx.doi.org/10.1002/cncr.11175

[15] Renshaw, A.A. and Gould, E.W. (2002) Why There Is the Tendency to "Overdiagnose” the Follicular Variant of Papillary Thyroid Carcinoma. American Journal of Clinical Pathology, 117, 19-21. http://dx.doi.org/10.1309/CJEU-XLQ7-UPVE-NWFV

[16] Rosai, J., Carcangiu, M.L. and Delellis, R.A. (1992) Follicular Carcinomas, Papillary Carcinomas. In: Rosai, J., Sobin, J. and Sobin, L.H., Eds., Tumors of Thyroid Gland, Atlas of Tumor Pathology, Armed Forces Institute of Pathology, Washington DC, 49-121.

[17] Rivera, M., Tuttle, R.M. and Patel, S., et al. (2009) Encapsulated Papillary Thyroid Carcinoma: A Clinic-Pathologic Study of 106 Cases with Emphasis on Its Morphologic Subtypes (Histologic Growth Pattern). Thyroid, 19, 119-127. http://dx.doi.org/10.1089/thy.2008.0303

[18] Fusco, A., Chiappetta, G., Hui, P., et al. (2002) Assessment of RET/PTC Oncogene Activation and Clonality in Thyroid Nodules with Incomplete Morphological Evidence of Papillary Carcinoma: A Search for the Early Precursors of Papillary Cancer. American Journal of Pathology, 160, 2157-2167. http://dx.doi.org/10.1016/S0002-9440(10)61164-9

[19] Barroeta, J.E., Baloch, Z.W., Lal, P., et al. (2006) Diagnostic Value of Differential Expression of CK19, Galectin-3, HMBE-1, ERK, RET, and p16 in Benign and Malignant Follicular Derived Lesions of the Thyroid: An Immunohistochemical Tissue Microarray Analysis. Endocrine Pathology, 17, 225-234. http://dx.doi.org/10.1385/EP:17:3:225

[20] Shahebrahimi, K., Madani, S.H., Fazaeli, A.R., Khazaei, S., et al. (2013) Diagnostic Value of CD56 and nm23 Markers in Papillary Thyroid Carcinoma. Indian Journal of Pathology and Microbiology, 56, 2-5. http://dx.doi.org/10.4103/0377-4929.116139

[21] Salajegheh, A., Pectu, E.B., Smith, R.A. and Lam, A.K.Y. (2008) Follicular Variant of Papillary Thyroid Carcinoma: A Diagnostic Challenge for Clinicians and Pathologists. Postgraduate Medical Journal, 84, 78-82. http://dx.doi.org/10.1136/pgmj.2007.064881

[22] Adeniran, A.J., Zhu, Z., Gandhi, M., Steward, D.L., Fidler, J.P., Giordano, T.J., Biddinger, P.W. and Nikiforov, Y.E. (2006) Correlation between Genetic Alterations and Microscopic Features, Clinical Manifestations, and Prognostic Characteristics of Thyroid Papillary Carcinomas. American Journal of Surgical Pathology, 30, 216-222. http://dx.doi.org/10.1097/01.pas.0000176432.73455.1b 